Research article

\title{
Mycobacterial and nonbacterial pulmonary complications in hospitalized patients with human immunodeficiency virus infection: A prospective, cohort study Bekele Afessa MD,2
}

Address: 1Division of Pulmonary and Critical Care Medicine, Department of Medicine, University of Florida Health Science Center,
Jacksonville, Florida, USA and 2Division of Pulmonary and Critical Care Medicine and Internal Medicine, Mayo Clinic, 200 First Street SW,
Rochester, MN 55905, USA E-mail: afessa.bekele@mayo.edu

Published: 19 September 2001

BMC Pulmonary Medicine 200I, I:I
Received: 22 June 2001

Accepted: 19 September 2001

This article is available from: http://www.biomedcentral.com/I47/-2466/I/I

(C) 200I Afessa; licensee BioMed Central Ltd. Verbatim copying and redistribution of this article are permitted in any medium for any non-commercial purpose, provided this notice is preserved along with the article's original URL. For commercial use, contact info@biomedcentral.com

\begin{abstract}
Background: A prospective observational study was done to describe nonbacterial pulmonary complications in hospitalized patients with human immunodeficiency virus (HIV) infection.

Methods: The study included I,225 consecutive hospital admissions of 599 HIV-infected patients treated from April 1995 through March 1998. Data included demographics, risk factors for HIV infection, Acute Physiology and Chronic Health Evaluation (APACHE) II score, pulmonary complications, $\mathrm{CD}^{+}$lymphocyte count, hospital stay and case-fatality rate.

Results: Patient age (mean \pm SD) was $38.2 \pm 8.9$ years, $62 \%$ were men, and $84 \%$ were African American. The median APACHE II score was 14 , and median CD4 $4^{+}$lymphocyte count was $60 / \mu \mathrm{L}$. Pulmonary complications were Pneumocystis carinii pneumonia (85) in 78 patients, Mycobacterium avium complex (5I) in 38, Mycobacterium tuberculosis (40) in 35, Mycobacterium gordonae (II) in II, Mycobacterium kansasii (10) in 9, Cytomegalovirus (10) in 10, Nocardia asteroides (3) in 3, fungus ball (2) in 2, respiratory syncytial virus (I), herpes simplex virus (I), Histoplasma capsulatum (I), lymphoma (3) in 3, bronchogenic carcinoma (2) in 2, and Kaposi sarcoma (I). The case-fatality rate of patients was 11\% with Pneumocystis carinii pneumonia; 5\%, Mycobacterium tuberculosis; 6\%, Mycobacterium avium complex; and 7\%, noninfectious pulmonary complications.
\end{abstract}

Conclusion: Most pulmonary complications in hospitalized patients with HIV are from Pneumocystis and mycobacterial infection.

\section{Background}

Since the beginning of the acquired immunodeficiency syndrome (AIDS) epidemic, pulmonary complications have been major causes of morbidity and mortality in patients with human immunodeficiency virus (HIV) infection [1]. In addition to the common pulmonary diseases affecting immunocompetent individuals, HIV seroposi- tive patients are prone to other infectious and noninfectious complications. The types of pulmonary complications that develop depend on the degree of immunosuppression, HIV transmission category, and geographic location. Antiretroviral therapy and Pneumocystis carinii pneumonia (PCP) prophylaxis have improved the clinical course and outcome of HIV- 
infected patients. However, pulmonary complications, including PCP, have remained common [2].

The Pulmonary Complications, Intensive Care Unit Support, and Prognostic Factors of Hospitalized Patients With HIV (PIP) Study is a single-center, prospective observational study designed to describe the types of pulmonary complications and the role of critical care support and to determine the prognostic factors of hospitalized HIV-infected adults. Knowledge of the types of pulmonary complications in patients with HIV infection will help clinicians to develop better diagnostic and therapeutic approaches. This article describes the nonbacterial pulmonary complications that were detected at hospital admission and during hospitalization of 599 patients.

\section{Material and Methods}

This prospective, observational study included 1,225 consecutive hospital admissions of 599 adults with HIV infection. The patients were identified from the hospital's computer system and admission logbooks and by contacting the case manager for patients with HIV. Patients 17 years or older with confirmed HIV infection were included in the study. All the patients were treated at the University Medical Center, Jacksonville, Florida, during the 3 years from April 1, 1995, through March 31, 1998. The University Medical Center is a 528-bed, teaching, inner-city hospital affiliated with the University of Florida. The study was approved by the Institutional Review Board of the hospital, which waived the need for informed consent.

Data were collected on age, sex, race, exposure category for HIV infection, $\mathrm{CD}_{4}^{+}$lymphocyte count, APACHE (Acute Physiology and Chronic Health Evaluation) II score, hospital stay, and in-hospital mortality. The investigator, a pulmonologist, reviewed all chest radiographs. All nonbacterial pulmonary complications that were diagnosed during patients' hospital stays were noted. The microbiologic, cytologic, and histologic findings of expectorated and induced sputum and other samples obtained by bronchoscopy, mediastinoscopy, and thoracotomy were noted.

Only confirmed pulmonary complications were included. $P$ carinii, mycobacterial, fungal, and viral pulmonary infections were diagnosed by identifying the organisms in stains or cultures of sputum, bronchial washing, bronchoalveolar lavage (BAL), and lung tissue. Multiple episodes of PCP and mycobacterial infections were defined by identifying the same organism on different admissions of the same patient. Fungus ball was diagnosed by the typical chest radiographic finding of a fungus ball in a preexisting cavity. Acute asthma exacerbation, acute exacerbation of chronic obstructive pulmonary disease (COPD), and cardiogenic pulmonary edema were diagnosed by physical findings in the appropriate clinical setting. Pulmonary embolism was documented by pulmonary angiogram or spiral computed tomography of the chest. Pulmonary contusion was diagnosed by the development of radiographic densities of the chest following chest trauma. Pulmonary sarcoidosis and malignancies were diagnosed by bronchoscopy. In patients with Mycobacterium tuberculosis (MTB) infection, antibiotic susceptibility test results were reviewed.

For the purpose of this study, multiple hospital admissions of the same patient were analyzed independently of each other. StatView 5.0 computer software (SAS Institute Inc., Cary, NC) was used for statistical analysis. All means were expressed with their standard deviations (SDs). Comparisons between groups were made by using the Student $t$, Mann-Whitney $\mathrm{U}, \mathrm{X}^{2}$, and Fisher exact tests. $P$ values $<0.05$ were considered significant. Continuity correction was used to determine the $P$ values of $\mathrm{X}^{2}$ tests. The sensitivity, specificity, and positive and negative predictive values of lactate dehydrogenase (LDH) for the diagnosis of PCP were calculated by using the normal high serum LDH level for our laboratory of 210 units $/ \mathrm{mL}$ as a cutoff point.

\section{Results}

Demographic characteristics, exposure categories, $\mathrm{CD}_{4}^{+}$ lymphocyte count, and APACHE II scores of the hospital admissions are listed in Table 1 . The primary reason for hospitalization was pulmonary in 356 of the 1,225 hospital admissions (29\%). The nonbacterial pulmonary complications observed during the study are listed in Table 2. The median APACHE II score of patients with PCP, MTB, and $M$ avium complex (MAC) were 13,15 , and 16 , respectively.

PCP was diagnosed in $85(7 \%)$ of the 1,225 hospital admissions ( 78 patients). Seventeen of the 345 admissions (5\%) during the first year of the study had PCP compared with 29 of 435 admissions (7\%) during the second and 39 of 445 admissions (9\%) during the third period ( $P=$ 0.1050). Two episodes of PCP were diagnosed in 7 and a single episode in 71 patients during the study. In the $7 \mathrm{pa}-$ tients who had 2 episodes of PCP, the time between the episodes ranged from 2 to 24 months. Induced sputum from 288 hospital admissions and BAL from 69 hospital admissions were examined for $P$ carinii by using monoclonal antibodies. The methods used to diagnose PCP are listed in Table 3. Seventy-seven of the 85 hospital admissions with PCP had induced sputum examined for $P$ carinii; 62 (81\%) were positive. PCP was diagnosed by BAL in 15 hospital admissions with negative findings from induced sputum. Induced sputum was diagnostic of PCP in 
2 patients whose BAL results were negative. $P$ carinii involved a cervical lymph node in 1 patient.

Table I: The Demographic Characteristics, Exposure Categories, CD4 ${ }^{+}$Lymphocyte Count, and APACHE II Scores of I,225 Hospital Admissions of 599 Patients With HIV. *

\begin{tabular}{lc}
\hline Variable & Result \\
\hline & \\
Age (mean \pm SD) $(y)$ & $38.2 \pm 8.9$ \\
Sex (no. and \% of admissions) & $754(62)$ \\
Male & $471(38)$ \\
Female & \\
Race (no. and \% of admissions) & $1,026(84)$ \\
African American & $190(16)$ \\
White & $9(1)$ \\
Hispanic & \\
Exposure category (no. and \% of admissions) & $333(27)$ \\
Injection drug use & $194(16)$ \\
Heterosexual contact & $138(11)$ \\
Homosexuality & $32(3)$ \\
Commercial sex work \& injection drug use & $30(2)$ \\
Homosexuality \& injection drug use & $29(2)$ \\
Commercial sex work & $22(2)$ \\
Blood transfusion & 1 \\
Needle stick & $446(36)$ \\
Unidentified & $159 \pm 223(60)$ \\
CD4 $4^{+}$lymphocyte count/ $\mu \mathrm{L}$ (median) & $15 \pm 7(14)$ \\
APACHE II score (median) &
\end{tabular}

*APACHE = Acute Physiology and Chronic Health Evaluation; HIV = human immunodeficiency virus.

Table 2: Nonbacterial Pulmonary Complications in I,225 Hospital Admissions.*

\begin{tabular}{lc}
\hline Complication & Admissions (no.) \\
\hline & \\
Infectious & 115 \\
Mycobacterial infections & 85 \\
Pneumocystis carinii pneumonia & 13 \\
Viral infections & 3 \\
Nocardia asteroides & 2 \\
Fungus ball & 1 \\
Histoplasma capsulatum & \\
Noninfectious & 10 \\
Acute asthma exacerbation & 7 \\
Cardiogenic pulmonary edema & 6 \\
Malignancies & 3 \\
Sarcoidosis & 2 \\
Pulmonary embolism & 1 \\
Acute exacerbation of COPD & 1 \\
Traumatic pulmonary contusion & \\
\hline
\end{tabular}

${ }^{*} \mathrm{COPD}=$ chronic obstructive pulmonary disease; HIV = human immunodeficiency virus.
Serum LDH was measured in 200 hospital admissions in which the primary diagnosis was pneumonia: $57 \mathrm{PCP}$ and 143 non-PCP. Among these 200 hospital admissions, the sensitivity, specificity, and negative and positive predictive values of serum $\mathrm{LDH}$ in the diagnosis of PCP were $98 \%, 31 \%, 98 \%$, and $36 \%$, respectively.

Table 3: Methods Used to Diagnose Pneumocystis carinii Pneumonia in 85 Hospital Admissions.

\begin{tabular}{lc}
\hline Diagnostic method & Admissions (no.) \\
\hline & \\
Induced sputum & 61 \\
Bronchoalveolar lavage & 21 \\
Open lung biopsy & 2 \\
Bronchoalveolar lavage and induced sputum & 1 \\
\hline
\end{tabular}

The initial chest radiograph obtained at hospital admission showed no infiltrate in 5 , focal infiltrate in 7 , and diffuse infiltrate in 73 of the 85 hospital admissions with $\mathrm{PCP}$. The differences in $\mathrm{CD}_{4}^{+}$lymphocyte count, duration of hospital stay, and mortality of patients with and without PCP are listed in Table 4. The $\mathrm{CD}_{4}^{+}$lymphocyte count of 5 patients with PCP was $>200 / \mu \mathrm{L}$. Pulmonary mycobacterial coinfection was identified in 18 of the 85 hospital admissions with PCP (21\%). Cytomegalovirus (CMV) was isolated from the BAL of 7 hospital admissions with PCP.

Mycobacteria were isolated from the respiratory specimen of 115 hospital admissions. The frequency of acidfast bacilli (AFB) smear in culture-positive MTB and MAC respiratory infection is listed in Table 5. Multiple episodes of pulmonary mycobacterial infections were diagnosed in 11 patients during the 3-year study: 3 episodes of pulmonary tuberculosis in 1 patient and 2 episodes in 3 patients; 5 episodes of pulmonary MAC in 2 patients, 3 in 1 patient, and 2 in 3 patients; and 2 episodes of pulmonary $M$ kansasii in 1 patient. The time between the different episodes ranged from 2 weeks to 2 years. MTB was isolated from respiratory specimens in 40 hospital admissions of 35 patients. Thirteen of the 345 admissions (4\%) during the first year of the study had respiratory MTB infection compared with 13 of 435 admissions (3\%) during the second and 14 of 445 admissions $(3 \%)$ during the third period $(P=0.8180)$. All the MTB isolates were sensitive to isoniazid. Rifampin resistance developed in 1 patient during the second episode of MTB infection.

MAC was isolated from respiratory specimens in 51 hospital admissions of 38 patients. Eighteen of the $345 \mathrm{ad}-$ 
Table 4: Differences in CD4 ${ }^{+}$Lymphocyte Count, Duration of Hospital Stay, and Mortality Between Patients With and Without Pneumocystis Carinii Pneumonia.*

\begin{tabular}{lccc}
\hline Variable & PCP & No PCP \\
\hline CD4+ lymphocytes (no./ $\mu \mathrm{L})$ (median) & $58 \pm 95(30)$ & $168 \pm 228(70)$ & $<0.0001$ \\
Duration of hospital stay (days) (median) & $10.2 \pm 9.2(8)$ & $5.9 \pm 6.3(4)$ & $<0.0001$ \\
Mortality (\%) & 11 & 5 & 0.0568 \\
\hline
\end{tabular}

${ }^{*} \mathrm{PCP}=$ Pneumocystis carinii pneumonia.

Table 5: Frequency of Negative Acid-Fast Bacilli Smear and Extrapulmonary Involvement.*

\begin{tabular}{lcc}
\hline & \multicolumn{2}{c}{ Incidence } \\
\cline { 2 - 3 } Variable & MTB & MAC \\
& & \\
\hline & & \\
Sputum AFB smear negative & $15 / 35(43 \%)$ & $38 / 45(84 \%)$ \\
BAL AFB smear negative & $4 / 8(50 \%)$ & $6 / 6(100 \%)$ \\
Extrapulmonary involvement & & \\
$\quad$ Blood & 4 & 27 \\
Gastrointestinal tract & 5 & 26 \\
Bone marrow & 1 & 1 \\
$\quad$ Lymph node & 4 & - \\
Cerebrospinal fluid & 3 & \\
Soft tissue & 2 & \\
& & \\
\hline
\end{tabular}

*AFB = acid-fast bacilli; BAL = bronchoalveolar lavage; $M A C=$ Mycobacterium avium complex; MTB = Mycobacterium tuberculosis.

missions (5\%) during the first year of the study had respiratory MAC infection compared with 16 of 435 admissions (4\%) during the second and 17 of 445 admissions $(4 \%)$ during the third period $(P=0.5095)$. Lung tissue confirming the diagnosis was obtained in only 1 of the patients with MAC. $M$ gordonae was isolated from respiratory specimens in 11 hospital admissions of 11 patients and $M$ kansasii in 10 hospital admissions of 9 patients. Nontuberculous mycobacteria were identified in 3 patients.

The median $\mathrm{CD}_{4}^{+}$lymphocyte count in the hospital admissions with MTB was $94 / \mu \mathrm{L}$ compared with $10 / \mu \mathrm{L}$ in those with MAC $(P<0.0001)$. The initial chest radiograph at hospital admission showed no infiltrate in 10 hospital admissions with pulmonary MTB and in 17 hospital admissions with pulmonary MAC. The extrapulmonary sites from which MTB and MAC were isolated are listed in Table 5 . The in-hospital case-fatality rate of hospital admissions with pulmonary MTB was $5 \%$ (2 of 40) compared with $6 \%$ (3 of 51) of those with pulmonary MAC $(P>0.9999)$.

Viral infections were detected in the BAL in 13 hospital admissions among 13 different patients: CMV in 10, respiratory syncytial virus in 1 , herpes simplex virus in 1 , and influenza $B$ virus in 1 . Of the 13 hospital admissions with viral infection, only 1 patient, who had CMV, died. Pulmonary involvement by Nocardia asteroides was seen in 3 patients, none of whom died. Fungus ball was seen on the chest radiograph of 2 patients, 1 of whom died. Pulmonary histoplasmosis was diagnosed in a patient of South American origin who died during hospitalization.

Malignancies involving the lungs were detected in 6 hospital admissions of 6 different patients: 2 bronchogenic carcinoma, 2 non-Hodgkin lymphoma, 1 Hodgkin lymphoma, and 1 Kaposi sarcoma. The 2 patients with bronchogenic carcinoma were 48- and 52-year-old AfricanAmerican men who smoked cigarettes. The risk factor for HIV transmission was homosexuality in 1 patient with small cell cancer and injection drug use in the other patient with squamous cell cancer. Of the 6 patients with malignancy, only 1 , who had Hodgkin lymphoma, died in the hospital. All 3 patients with pulmonary sarcoidosis were of African-American origin and survived to hospital dismissal. Ten patients were hospitalized for acute asthma attack, and all survived to hospital dismissal. Of the patients admitted for cardiogenic pulmonary edema, pulmonary embolism, acute exacerbation of COPD, and traumatic pulmonary contusion, only 1 patient, who had cardiogenic pulmonary edema, died before hospital dismissal.

\section{Discussion}

This study describes the nonbacterial pulmonary complications detected among 1,225 hospital admissions of 599 adult patients with HIV infection. The study population consisted predominantly of African Americans, with a significant number of heterosexual and unidentified transmission categories. The HIV transmission category 
influences the types of pulmonary complications that develop in patients with HIV. Because homosexuality and injection drug use are the major HIV transmission categories in the United States, the findings in our study may not apply to other countries.

PCP was the most common nonbacterial pulmonary complication caused by a single organism in our study. Despite its decline as a result of widespread antiretroviral therapy and chemoprophylaxis, PCP has remained a common complication in patients with HIV infection $[2,3]$. In a prospective multicenter study of HIV seropositive patients, for $79 \%$ of those with a $\mathrm{CD} 4^{+}$lymphocyte count $<200 / \mu \mathrm{L}$ receiving PCP chemoprophylaxis, the rate of first episode PCP was 3.3 per 100 person-years [4]. Although actual data were not available, high rates of noncompliance with and lack of access to antiretroviral therapy and chemoprophylaxis were likely in our study population, which included a significant number of medically underserved patients without perceived risk for HIV.

Previous studies addressed the need for simpler tests and strategies to identify patients with a high probability of having PCP to initiate either empiric treatment or aggressive investigation $[5,6]$. These studies reported that an increase in LDH value had variable specificity and sensitivity for the diagnosis of PCP. HIV seropositive patients are at increased risk for PCP when their $\mathrm{CD} 4^{+} \mathrm{lym}-$ phocyte count is $<200 / \mu \mathrm{L}$. Similar to the multicenter study by the Pulmonary Complications of HIV Infection Study Group [4], 94\% of the patients with PCP had a $\mathrm{CD} 4^{+}$lymphocyte count $<200 / \mu \mathrm{L}$ in the PIP study. The classic chest radiographic findings of patients with PCP include bilateral diffuse opacities [7]. However, some patients present with unusual chest radiographic findings $[7,8]$. In a multicenter study from the United States, the in-hospital mortality rate of hospitalized patients with cytologically confirmed PCP was 14\% [9]. The PIP study showed that PCP is unusual in patients with normal $\mathrm{CD}_{4}^{+}$lymphocyte count and serum LDH concentration, can present with atypical chest radiographic findings, can be diagnosed from induced sputum in most of the cases, and is associated with $11 \%$ mortality.

In the PIP study of predominantly African-American patients, nearly $50 \%$ of the MTB isolates had a negative AFB smear, none of the isolates was isoniazid resistant, and $25 \%$ of the admissions with MTB respiratory infection had no infiltrate on the initial chest radiograph. HIV-infected individuals are highly susceptible to contracting tuberculous infection and are prone to rapid evolution of active disease [10]. The rate of tuberculosis is high in African Americans and in injection drug users [11]. Although the prevalence of a positive AFB smear in patients with pulmonary MTB is approximately the same in HIV-infected and noninfected individuals, it may decline in advanced HIV-mediated immunosuppression [12]. A previous study of 133 HIV-infected adults with pulmonary MTB showed 14\% of them had a normal chest radiograph [13].

The isolation of MAC in BAL or sputum does not distinguish between disease and colonization. Prospective cohort studies have shown that in $25 \%$ to $35 \%$ of patients with HIV infection disseminated MAC disease eventually develops $[14,15]$. The risk of disseminated MAC disease depends on the severity of the immunodeficiency. In the PIP study, patients with MAC respiratory isolates had a low $\mathrm{CD} 4^{+}$lymphocyte count, and the majority of them had evidence of disseminated disease.

CMV is the most common opportunistic viral infection in patients with HIV [16]. Although CMV was isolated from the BAL of 10 patients in the PIP study, its isolation does not distinguish between infection and colonization. Fungal infections are common in patients with HIV infection. HIV-infected individuals residing in areas endemic for Histoplasma capsulatum are at increased risk for $H$ capsulatum infection [17]. Histoplasmosis is uncommon in individuals from areas in which $H$ capsulatum is nonendemic and usually represents reactivation [18]. The PIP study was performed in an area in which $H$ capsulatum is nonendemic, and there was only 1 case of pulmonary histoplasmosis in a patient who had resided in an area endemic for $H$ capsulatum. Although the lungs are the primary site of cryptococcal infection, pulmonary disease was believed to be uncommon in patients with AIDS [19]. No pulmonary cryptococcal disease was diagnosed in the PIP study. However, a recent review of 48 autopsies of HIV-infected patients from the same institution showed cryptococcal pneumonia to be as frequent as PCP [20].

In patients with HIV infection, about 20\% are expected to develop a malignancy [21]. The PIP study population is predominantly African American. Blacks with HIV infection have a lower risk of cancer developing than nonblacks [22]. Kaposi sarcoma and non-Hodgkin lymphoma are the most common malignancies, and they are epidemiologically linked to HIV. Kaposi sarcoma has been reported in all HIV risk groups. However, it occurs predominantly in homosexual and bisexual men [23]. In the PIP study, homosexuality was not a predominant HIV risk factor, and pulmonary Kaposi sarcoma was diagnosed in only 1 patient.

Conflicting evidence links lung cancer with HIV infection. At the beginning of the AIDS epidemic, few cases of bronchogenic carcinoma were reported [24,25]. Using 
the registry of Bellevue Hospital in New York City, Chan et al. [26] showed no link between lung cancer and HIV infection. Another population-based study from San Francisco showed no excess of lung cancer among a cohort of men with AIDS [27]. The Italian Cooperative Study Group described 19 cases of HIV-associated lung cancer -16 in tobacco smokers [28]. More recent studies have indicated an increase in incidence of primary lung cancer in patients with HIV infection [22,29]. Although there was no case of adenocarcinoma in the PIP study, it is the predominant cell type of bronchogenic carcinoma in patients with HIV infection [22,26,30]. The Pulmonary Complications of HIV Infection Study Group has shown upper respiratory infection and bronchitis to be common in patients with HIV infection [3]. Because the PIP study included only hospitalized patients, minor respiratory problems were likely to have been ignored.

The PIP study has several limitations. Some important data, such as smoking history and the use of chemoprophylaxis and antiretroviral drugs, were not collected. No attempt was made to identify patients with pulmonary hypertension. The diagnostic approaches varied according to the patients' primary physicians. The investigator's unblinded review of chest radiographs may have introduced biases into the study. Diagnostic fiberoptic bronchoscopy was performed in only 69 hospital admissions, and thoracotomy and mediastinoscopy were rarely required. This approach may have led to underdiagnosis of some of the pulmonary complications. Although MAC and CMV were isolated from the sputum and BAL of many patients, the presence of disease could not be ascertained because tissues were not available for examination. Because the PIP study was started before highly active antiretroviral treatment was introduced, the findings may not apply now.

\section{Conclusions}

The PIP study describes the nonbacterial pulmonary complications in 1,225 hospital admissions of 599 patients with HIV infection. The present data confirm previously described findings addressing these complications [2-7],[11-15,22]. With the advent of antiretroviral therapy and chemoprophylaxis against some of the opportunistic infections, the morbidity and mortality of patients with HIV infection have declined in the last decade. The study showed that PCP is still a frequent complication, despite the availability of chemoprophylaxis. Although pulmonary tissue involvement is not confirmed, most of the patients with MAC isolated from their sputum or BAL have disseminated disease. These findings suggest the need for more effective preventive measures in similar patient populations. The application of highly active antiretroviral treatment and the development of more effective chemoprophylaxis against opportunistic infections are likely to be associated with changes in the types and severity of pulmonary complications that we see in these patients. The pulmonary complications are also likely to vary according to geographic locations, HIV risk factor, and severity of immunosuppression. To keep up with these changes and variations, we must continue to monitor and to describe the pulmonary complications that develop in patients with HIV infection.

\section{Competing interests}

None declared.

\section{Acknowledgment}

I thank Dr. David L. Armbruster for reviewing this manuscript.

\section{References}

I. Murray JF, Felton CP, Garay SM, Gottlieb MS, Hopewell PC, Stover $D E$, Teirstein AS: Pulmonary complications of the acquired immunodeficiency syndrome. Report of a National Heart, Lung, and Blood Institute workshop. N Engl J Med 1984, 3 1 0:1682-1688

2. Moore RD, Chaisson RE: Natural history of opportunistic disease in an HIV-infected urban clinical cohort. Ann Intern Med 1996, I 24:633-642

3. Wallace JM, Hansen NI, Lavange L, Glassroth J, Browdy BL, Rosen MJ, Kvale PA, Mangura BT, Reichman LB, Hopewell PC: Respiratory disease trends in the Pulmonary Complications of HIV Infection Study cohort. Pulmonary Complications of HIV Infection Study Group. Am J Respir Crit Care Med 1997, I 55:72-80

4. Stansell JD, Osmond DH, Charlebois E, LaVange L, Wallace JM, Alexander BV, Glossroth J, Kvale PA, Rosen MJ, Reichman LB, et al: Predictors of Pneumocystis carinii pneumonia in HIV-infected persons. Am J Respir Crit Care Med I 997, I 55:60-66

5. Zaman MK, White DA: Serum lactate dehydrogenase levels and Pneumocystis carinii pneumonia. Diagnostic and prognostic significance. Am Rev Respir Dis 1988, I37:796-800

6. Boldt MJ, Bai TR: Utility of lactate dehydrogenase vs radiographic severity in the differential diagnosis of Pneumocystis carinii pneumonia. Chest 1997, I I I: I I87- I I92

7. DeLorenzo LJ, Huang CT, Maguire GP, Stone DJ: Roentgenographic patterns of Pneumocystis carinii pneumonia in 104 patients with AIDS. Chest 1987, $91: 323-327$

8. Shin MS, Veal CF, Jessup JG, Ho KJ: Apical Pneumocystis carinii pneumonia in AIDS patients not receiving inhaled pentamidine prophylaxis. Chest I991, I00:| 462-|464

9. Bennett CL, Horner RD, Weinstein RA, Kessler HA, Dickinson GM, Pitrak DL, Gilman SC, George WL, Cohn SE, Simberkoff MS, et al: Empirically treated Pneumocystis carinii pneumonia in Los Angeles, Chicago, and Miami: 1987-1990. J Infect Dis 1995, 172:312-315

10. Daley CL, Small PM, Schecter GF, School GK, McAdam RA, Jacobs WR Jr, Hopewell PC: An outbreak of tuberculosis with accelerated progression among persons infected with the human immunodeficiency virus. An analysis using restriction-fragment-length polymorphisms. N Engl J Med 1992, 326:231-235

II. Given MJ, Khan MA, Reichman LB: Tuberculosis among patients with AIDS and a control group in an inner-city community. Arch Intern Med 1994, I 54:640-645

12. Klein NC, Duncanson FP, Lenox TH III, Pitta A, Cohen SC, Wormser GP: Use of mycobacterial smears in the diagnosis of pulmonary tuberculosis in AIDSIARC patients. Chest 1989, 95: I I90II92

13. Greenberg SD, Frager D, Suster B, Walker S, Stavropoulos C, Rothpearl A: Active pulmonary tuberculosis in patients with AIDS: Spectrum of radiographic findings (including a normal appearance). Radiology 1994, 193: II5-119

14. Hoover DR, Saah AJ, Bacellar H, Phair J, Detels R, Anderson R, Kaslow RA: Clinical manifestations of AIDS in the era of pneumocystis prophylaxis. Multicenter AIDS Cohort Study. N Engl J Med 1993, 329:1922-1926 
15. Katz MH, Hessol NA, Buchbinder SP, Hirozawa A, O'Malley P, Holmberg SD: Temporal trends of opportunistic infections and malignancies in homosexual men with AIDS. J Infect Dis 1994, I 70: 198-202

16. Smith MA, Brennessel DJ: Cytomegalovirus. Infect Dis Clin North Am 1994, 8:427-438

17. Wheat LJ, Slama TG, Zeckel ML: Histoplasmosis in the acquired immune deficiency syndrome. Am J Med 1985, 78:203-210

18. Salzman SH, Smith RL, Aranda CP: Histoplasmosis in patients at risk for the acquired immunodeficiency syndrome in a nonendemic setting. Chest I988, 93:916-921

19. Zuger A, Louie E, Holzman RS, Simberkoff MS, Rahal J]: Cryptococcal disease in patients with the acquired immunodeficiency syndrome. Diagnostic features and outcome of treatment. Ann Intern Med 1 986, 104:234-240

20. LaGatta M, Chiao J, Afessa B: Pulmonary complications in patients with HIV infection: Autopsy findings (abstract). Chest 1 997, I I 2 (Suppl): I 5S

21. Beral V, Jaffe H, Weiss R: Cancer surveys: Cancer, HIV and AIDS. Eur J Cancer I99I, 27: I057-1058

22. Johnson CC, Wilcosky T, Kvale P, Rosen M, Stansell J, Glassroth J, Reichman L, Wallace J, Markowitz N, Thompson JE, et al: Cancer incidence among an HIV-infected cohort. Pulmonary Complications of HIV Infection Study Group. Am J Epidemiol 1997, 1 46:470-475

23. Serraino D, Franceschi S, Tirelli U, Monfardini S: The epidemiology of acquired immunodeficiency syndrome and associated tumours in Europe. Ann Oncol 1992, 3:595-603

24. Braun MA, Killam DA, Remick SC, Ruckdeschel JC: Lung cancer in patients seropositive for human immunodeficiency virus. $R a-$ diology 1990, 175:34|-343

25. Monfardini S, Vaccher E, Pizzocaro G, Stellini R, Sinicco A, Sabbatani $S$, Marangolo M, Zagni R, Clerici M, Foa R, et al: Unusual malignant tumours in 49 patients with HIV infection. AIDS 1989, 3:449452

26. Chan TK, Aranda CP, Rom WN: Bronchogenic carcinoma in young patients at risk for acquired immunodeficiency syndrome. Chest 1993, 103:862-864

27. Reynolds P, Saunders LD, Layesfsky ME, Lemp GF: The spectrum of acquired immunodeficiency syndrome (AIDS)-associated malignancies in San Francisco, 1 980-1 987. Am J Epidemiol I 993, 137:19-30

28. Vaccher E, Tirelli U, Spina M, Errante D, Crosato I, Sabbatani S, Sinicco A, Monfardini S: Lung cancer in 19 patients with HIV infection. The Italian Cooperative Study Group on AIDS and Tumors. Ann Oncol 1993, 4:85-86

29. Parker MS, Leveno DM, Campbell TJ, Worrell JA, Carozza SE: AIDSrelated bronchogenic carcinoma: Fact or fiction? Chest 1998, I 13:154-|6|

30. Tenholder MF, Jackson HD: Bronchogenic carcinoma in patients seropositive for human immunodeficiency virus. Chest 1993, 104: 1049-1053

Publish with BioMed Central and every scientist can read your work free of charge

"BioMedcentral will be the most significant development for disseminating the results of biomedical research in our lifetime."

Paul Nurse, Director-General, Imperial Cancer Research Fund

Publish with BMC and your research papers will be:

- available free of charge to the entire biomedical community

- peer reviewed and published immediately upon acceptance

- cited in PubMed and archived on PubMed Central

- yours - you keep the copyright

Submit your manuscript here:

http://www.biomedcentral.com/manuscript/
BioMedcentral.com editorial@biomedcentral.com 